\title{
Wideband Tympanometry
}

\author{
Anirban Biswas ${ }^{1} \quad$ Nilotpal Dutta ${ }^{1}$ \\ ${ }^{1}$ Vertigo and Deafness Clinic, Kolkata, West Bengal, India
}

Ann Otol Neurotol ISO 2018;1:126-132
Address for correspondence Anirban Biswas, MBBS, DLO, Vertigo and Deafness Clinic, BJ-252, Salt Lake, Sector-2, Kolkata 700091, West Bengal, India (e-mail: vertigodeafnesstinnitus@gmail.com).

\begin{abstract}
Keywords

- middle ear

- traditional

tympanometry

- wideband

tympanometry

Traditional tympanometry done with $226 \mathrm{~Hz}$ probe tone frequency has some inherent defects and limitations due to which it does not give a very true picture of the middle ear status, and tympanometric findings are often very deceptive. It is not uncommon for otologists to find that on opening the middle ear, the pathology in the middle ear is very different from what they had expected from tympanometric findings. This is because the 226 or $220 \mathrm{~Hz}$, that is used for the traditional single tone tympanometry is based on physical convenience, i.e., practicalities in carrying out the test rather than on test performance, i.e., the diagnostic efficacy of the test. The $226 \mathrm{~Hz}$ tympanometry is a poor predictor of middle ear effusions in babies and will be wrong in approximately $50 \%$ of cases and diagnosis of ossicular chain discontinuity by type $A$ tympanogram is correct in only about $40 \%$ cases, if not lesser. In most cases of otosclerosis, though there is a middle ear stiffness, the tympanometric findings with traditional tympanometry show normal compliance, which is not expected in stiffness of the middle ear. Tympanometry is basically to identify common middle ear pathologies, such as middle ear effusion, ossicular chain discontinuity and otosclerosis. However, if in these very cases the diagnostic efficacy is so poor, then the objective of the test is lost. Traditional tympanometry has a lot of limitations and fallacies. These issues led scientists to sharpen the diagnostic efficacy of tympanometry and the final outcome of the research is wide band tympanometry (WBT). The special advantages of WBT, its difference from traditional single frequency tympanometry, and its clinical utility are presented in this article. Here, we review the concept of WBT, the basic mechanism, and its vast clinical applications. Most of the deficiencies of traditional tympanometry have been overcome by WBT. Not only that, the scope of tympanometry has been further widened by WBT and in addition to diagnosing middle ear pathologies with much more confidence, it can also be used for postoperative monitoring using non-pressurized wideband absorbance.
\end{abstract}

\section{Introduction}

Tympanometry is a test for evaluation of the conductivity of the middle ear. It assesses how efficiently the middle ear is transferring acoustic energy from the external auditory meatus to the inner ear. Different middle ear pathologies alter this function in diverse ways that are usually unique for the different middle ear pathologies. Tympanometry test can document how this function of transferring energy is altered and is hence used to diagnose the nature of the different middle ear disorders.
In tympanometry, a sound is presented to the external auditory meatus that strikes the eardrum and part of it gets reflected back into the external auditory meatus and part of it passes through the middle ear to the inner ear. The magnitude of the acoustic energy (sound) reflected off the eardrum into the external meatus (which can be recorded by a miniature very sensitive mic in the external meatus) depends on the stiffness of the middle ear; the more the stiffness, the more is the sound that is reflected back and the lesser is the sound transmitted through the middle ear to the inner ear. This stiffness of the middle ear can be mechanically varied 
by changing the air pressure in the external auditory meatus. The stiffness is least; that is, the middle ear is most compliant when the air pressure in the external auditory meatus is equal to that of the middle ear space. The best transfer of acoustic energy from the external to the inner ear takes place when this happens i.e., when the pressure in the middle ear space and that in the external auditory meatus is the same.

In tympanometry test, while the sound is presented to the ear, the air pressure of the external meatus is continually changed by an air pump, which, in turn, changes the stiffness of the middle ear. The actual stiffness, and hence the magnitude of the reflected sound, is dependent on the pathology in the middle ear and changes in different middle ear disorders. In different middle ear pathologies, this change in stiffness as a function of the change of air pressure varies uniquely. Hence, the acoustic energy reflected back from the eardrum in different grades of stiffness varies differently in different middle ear pathologies, and so by plotting the stiffness (or its opposite, i.e., the compliance or ease of transfer of acoustic energy) as a function of the changing air pressure, a graph is obtained, which is called the tympanogram. The tympanogram is different in different middle ear pathologies, and so it is used to diagnose different middle ear pathologies.

\section{Traditional Tympanometry}

Traditional tympanometry test is done at one particular frequency, usually $226 \mathrm{~Hz}$ (and uncommonly at 678, 800, and $1,000 \mathrm{~Hz}$ for special requirements). At $226 \mathrm{~Hz}$, the middle ear is stiffness dominated, and changes in stiffness of the middle ear is best ascertained with a $226-\mathrm{Hz}$ tone as most sounds of this particular frequency are reflected back without absorption when the middle ear is made most stiff (i.e., entire sound presented to the eardrum is reflected back into the external auditory meatus) with an external meatus pressure of +200 daPa. Hence only at $226 \mathrm{~Hz}$, compliance and admittance have a $1: 1$ relationship. Therefore, tympanometry with a $226-\mathrm{Hz}$ sound detects problems with the tympanic membrane and middle ear (tympanum) most easily, most simply, and with least complications. Traditional tympanometry evaluates the impedance or stiffness of the middle ear apparatus by calculating the energy that is reflected from the eardrum when a single pure tone sound (which is the probe tone frequency of $226 \mathrm{~Hz}$ ) impinges on it. The stiffer the middle ear, the more is the sound that is reflected off the eardrum, and by measuring the energy reflected off the eardrum, the impedance audiometer ascertains the stiffness of the middle ear. The sound absorbed by the middle ear and transmitted to the inner ear is not measured and documented in traditional tympanometry.

Traditional tympanometry has some shortcomings; for example, it does not tell us accurately whether there is some fluid in a type $C$ tympanogram. A type " $\mathrm{C}$ " tympanogram implies that there is no fluid in the middle ear though there is a negative middle ear pressure due to tubal dysfunction/ blockage. However, it is a common clinical experience that in many such cases with a type " $\mathrm{C}$ " tympanogram, there is a persistent heaviness in the ear and myringotomy in such a patient yields fluid that is not expected in a type "C" tympanogram, thereby belying the tympanometric finding. In persons with a perforation in the eardrum, no idea of the middle ear status is possible with traditional tympanometry; all that one gets is a flat tympanogram and there is no way to know whether the middle ear is otherwise normal or not. Sometimes there are artifacts or noise in the tympanogram especially in children, and repeating the tympanogram does not reduce the artifacts or noise. The $226-\mathrm{Hz}$ tone at which tympanometry is usually done is a poor predictor of middle ear effusions in babies and will be wrong in approximately $50 \%$ of cases (Liu et al 2014) whereas tympanogram with a $1000-\mathrm{Hz}$ tone is better in such cases but not perfect (Baldwin 2006). Therefore, there are a lot of limitations in traditional single-frequency reflectance tympanometry.

\section{Wideband Tympanometry}

An improvement on traditional/conventional tympanometry is the wideband tympanometry (WBT) that uses a wideband click stimulus $(226-8,000 \mathrm{~Hz})$ and not a single-frequency or pure-tone sound as the sound stimulus. WBT measures the middle ear's efficiency of sound transmission through the middle ear at multiple frequencies (instead of a single frequency) and hence evaluates middle ear function more completely. Different middle ear pathologies affect the transmission of sounds of different frequencies thorough the middle ear differently. Consequently, WBT overcomes many of the shortcomings and limitations of traditional single-frequency tympanometry.

Moreover, WBT measures not only the sound that is reflected off the eardrum (reflectance) as in traditional tympanometry but also the sound that passes into the middle ear across the eardrum (i.e., the sound that is absorbed by the middle ear called "absorbance"), and hence yields a lot more information about the middle ear conductive mechanism and its disorders. The impact of different middle ear pathologies on both reflectance and absorbance differs at multiple frequencies, and WBT allows us the advantage of documenting this difference very precisely. It is hence is a sharper diagnostic tool than traditional tympanometry. Just testing reflectance with a single frequency only, as is done in traditional tympanometry, does not yield all information about the functioning of the middle ear in different middle ear diseases. The plethora of data available about conductance or absorbance from WBT helps in the detection of middle ear problems with a much higher degree of accuracy than conventional tympanometry. WBT allows comprehensive analysis of middle ear status over a frequency range that includes the full speech spectrum from 226 to $8,000 \mathrm{~Hz}$ or more.

In essence, the difference between traditional and wideband tympanometry is (1) the stimulus and (2) the additional measurement of absorbance data. With WBT, a click is used that covers a frequency range from roughly $226 \mathrm{~Hz}$ up to (and if wanted even beyond) $8,000 \mathrm{~Hz}$. The time taken is the same as a traditional tympanometry test. WBT provides not only the 
same information as traditional tympanometry, that is, admittance, but also new information, that is, absorbance, and this it does at all perceivable frequencies across the speech spectrum. The middle ear is hence evaluated in much more detail,

The WBT output absorbance data are a three-dimensional (3D) graph (-Fig. 1) that factor in three variables or parameters, namely the middle ear pressure from +200 to $-600 \mathrm{daPa}$, the frequency from 200 to $8,000 \mathrm{~Hz}$ or more, and the absorbance in percentage.

However, from this 3D graph, different types of twodimensional (2D) graphs can be extracted. The software is capable of generating the normal traditional 2D single-frequency tympanogram with pressure and compliance at $226 \mathrm{~Hz}$ (-Fig. 2) or at any other desired frequency such as $687 \mathrm{~Hz}, 1,000 \mathrm{~Hz}$ (-Fig. 3), etc. It can also generate a $2 \mathrm{D}$ averaged wide band tympanogram ( - Fig. 4 ) with absorbance or compliance (y-axis) as a function of the pressure in daPa (x-axis), which is very useful in different middle ear disorders. It can also provide absorbance data as a $2 \mathrm{D}$ absorbance graphs at any desired pressure like at atmospheric or middle ear pressure. All these different outputs have clinically relevant diagnostic implications that are not available with traditional tympanometry. Wide band averaged tympanograms (by default $800-2,000 \mathrm{~Hz}$

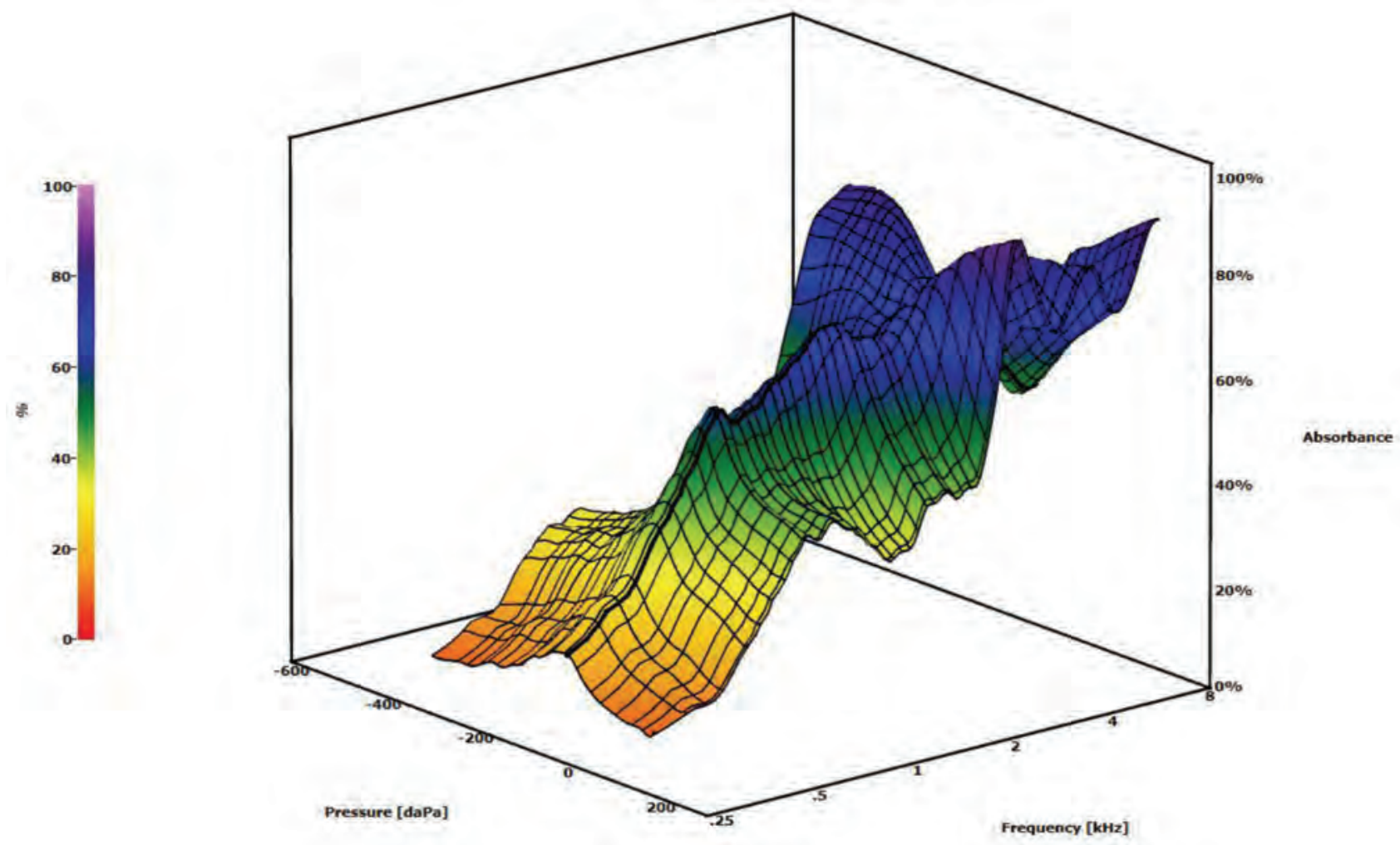

Fig. 1 Wide band tympanometry absorbance graph where the absorbance is plotted as a function of the middle ear pressure (+200 to -600 $\mathrm{daPa})$ as well as the frequency $(250-8,000 \mathrm{~Hz})$.

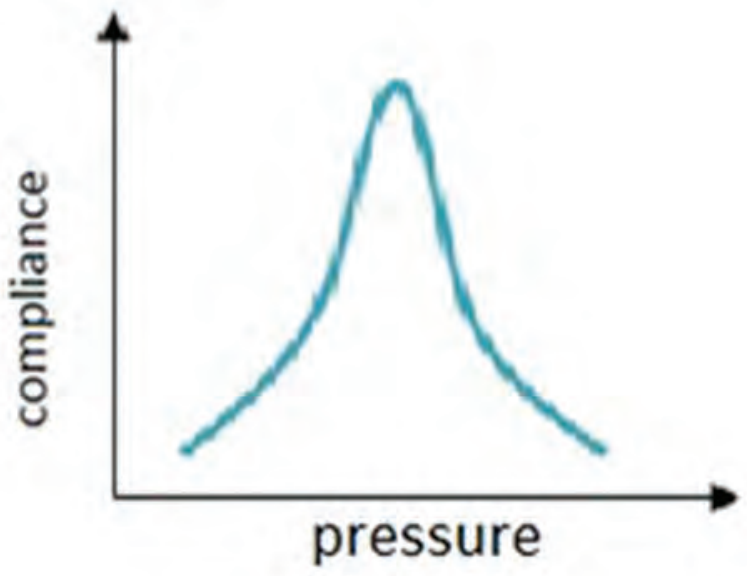

Fig. 2 The 226-Hz tympanogram-diagrammatic representation.

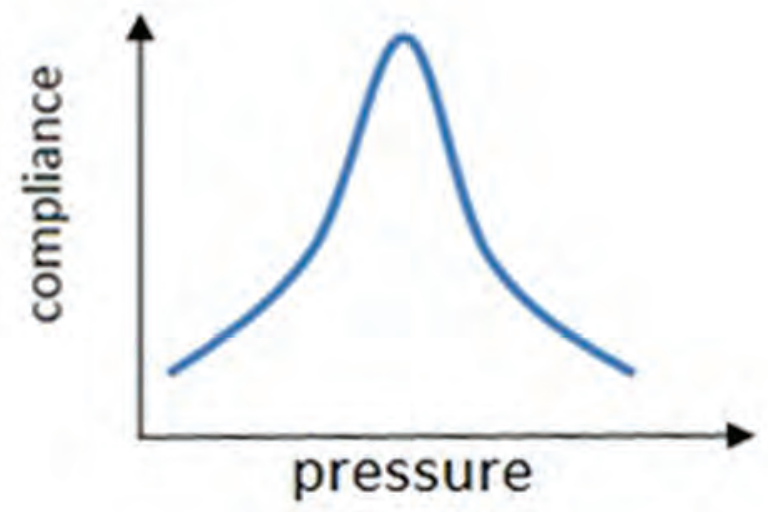

Fig. 3 The 1,000-Hz tympanogram-diagrammatic representation. 
in children and $375-2,000 \mathrm{~Hz}$ in adults) are much better detectors of middle ear effusions and are not contaminated by noise unlike the traditional tympanograms. Therefore, the scope of WBT is much wider than that of traditional tympanometry.

Individual, i.e., separate 2D graphs may be extracted from the WBT as in - Figs. 2, 3, 4, 5, 6, and $\mathbf{7}$.

The superiority of WBT over traditional tympanometry are manifold:

1. With WBT providing much more stable and robust recordings in children and in less cooperative patients, this is a definite advantage of it. In many patients, a very noisy graph and artifacts ( $\boldsymbol{- \text { Fig. }}$ 5) are obtained, which are very stubborn and repeatedly testing does not improve the recording quality. In such patients, a wide band averaged tympanogram is a big help, and a noise-free stable clear recording of the tympanogram is

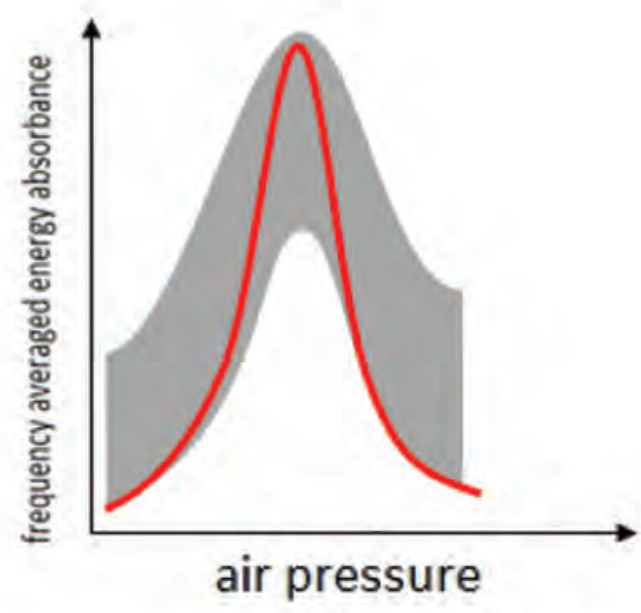

Fig. 4 Wideband averaged tympanogram.

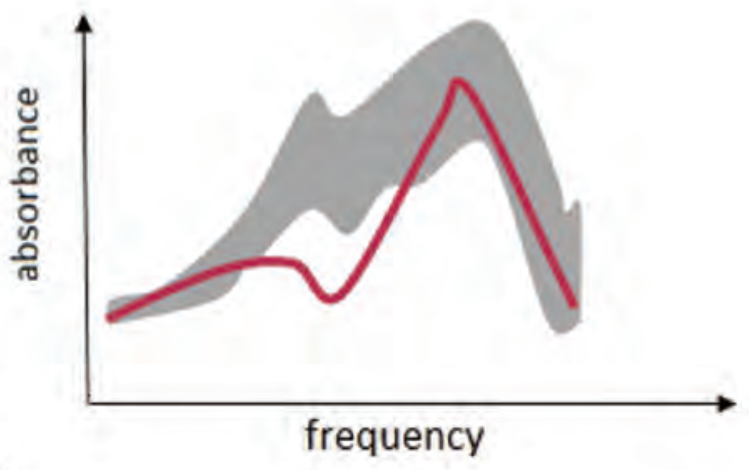

A

Fig. 6 (A) Diagrammatic representation of a 2D absorbance graph. (B) Diagrammatic representation of a 2D absorbance graph in a patient who has a type $C$ tympanogram in traditional tympanometry. obtained. Because of the averaging, the noise has almost no impact on the WBT.

2. Type " $\mathrm{C}$ " traditional tympanogram is interpreted as a eustachian tube-blocked negative middle ear pressure, air-filled tympanum without any fluid (as compliance is normal) in the middle ear. However, often there is a persistent heaviness in many such ears and many of such patients are subjected to a myringotomy that surprisingly reveals fluid and contradicts the tympanometric findings. This indicates that many patients with type C tympanogram have partially fluid-filled middle ears that cannot be diagnosed by traditional tympanometry. WBT is a big help in all such patients. WBT absorbance graph will show that at a particular range of frequencies, the absorbance is very low ( $\mathbf{- F i g .} \mathbf{6 B})$, but at another range of frequencies the absorbance is normal; this nearly flat absorbance graph with very little or low absorbance over a range of frequencies is an indication that there must be some fluid in the middle

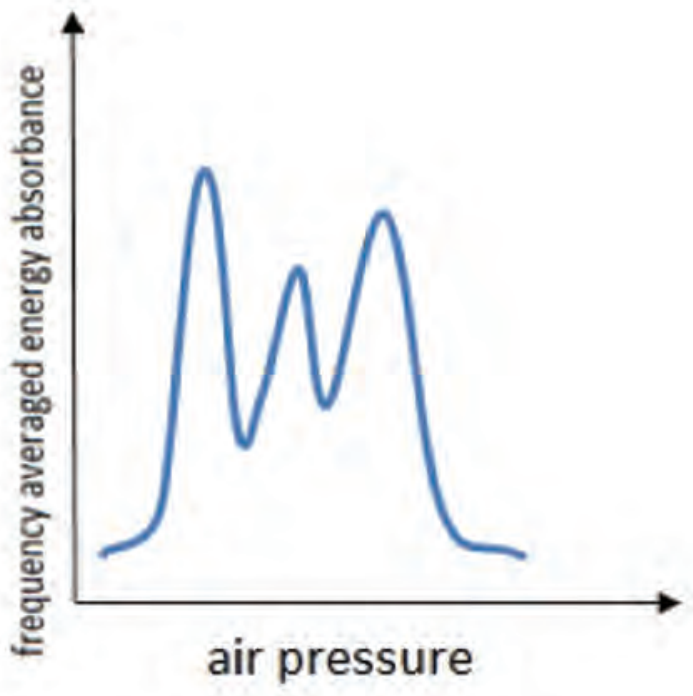

Fig. 5 Noisy $1000-$ Hz traditional tympanogram with lots of artifacts.

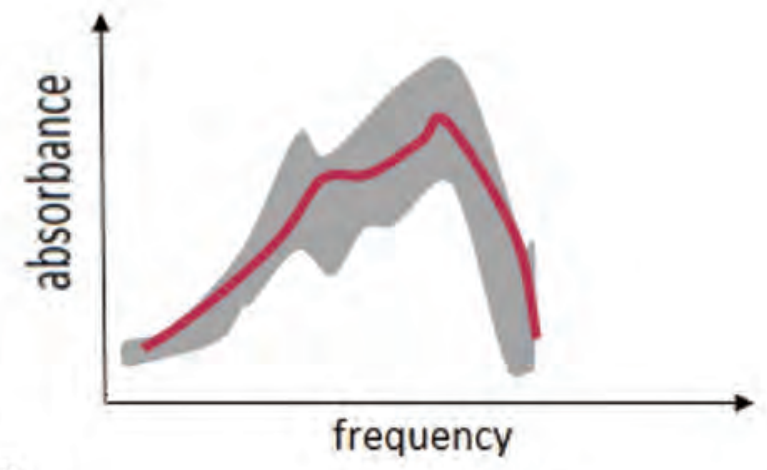

B 


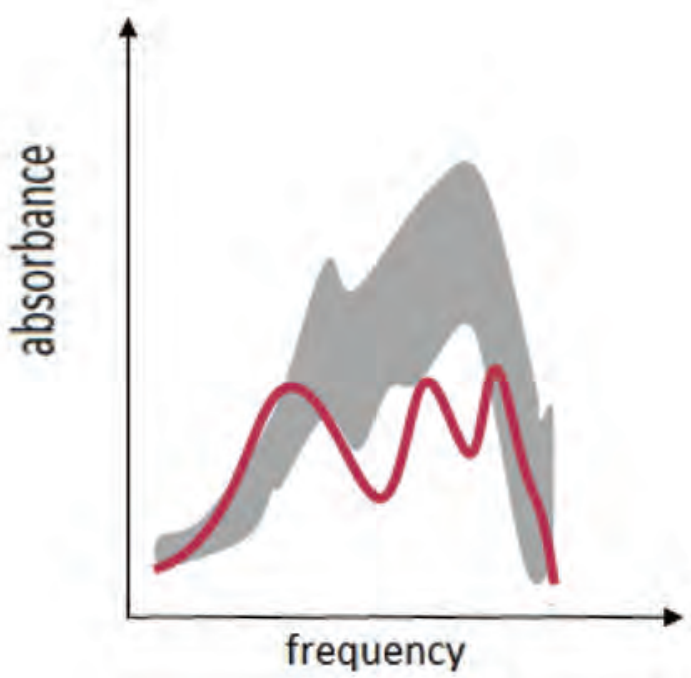

Fig. 7 Wide band tympanogram absorbance graph in two-dimensional with the absorbance in percentage in the $y$-axis and the frequency from 250 to $8,000 \mathrm{~Hz}$ in the $\mathrm{x}$-axis.

ear, and hence a myringotomy is warranted. The traditional tympanogram done at a single frequency will not show this very low compliance over a particular range of frequencies, and it will show normal or near-normal compliance, because at another frequency range, the compliance is normal. However, if in the WBT test the compliance is normal (-Fig. $\mathbf{6 B}$ ) in all frequency ranges, it would indicate that there is no fluid in the tympanum.
3. Wide band tympanometry allows the clinician to assess the middle ear condition even if there is a perforation in the eardrum or if there is a patent grommet in the eardrum; this is not possible with traditional tympanometry. If the middle ear function is normal, WBT absorbance graph will show a tracing that more or less tallies with the normative data with a peak usually below or around $1,000 \mathrm{~Hz}$, maybe at other places also, but there will be a peak somewhere in the frequency range $(200-8,000 \mathrm{~Hz})$ with a close match to the normative data. However, if the middle ear is moist or abnormal in other ways, the absorbance graph will be more or less flat and not tally with the normative graph.

\section{Information Derived from Wideband Tympanometry at the Press of a Button}

Ease of operation is as good as the traditional tympanometry, and though a huge lot of information about the functionality of the middle ear can be extracted from WBT, there is nothing extra that the operator has to perform while carrying out the test. The operator just has to ensure an air-tight seal and press a single button for the complete test to be done in a few seconds. WBT system offers three different types of outputs, namely (1) 3D WBT view ( - Fig. 1), (2) traditional 2D tympanogram at any selected frequency (-Figs. 2, 3), and (3) 2D absorbance graph (-Fig. 4). Admittance tympanograms are available for probe tone frequencies $226 \mathrm{~Hz}, 678 \mathrm{~Hz}, 800 \mathrm{~Hz}$, and $1,000 \mathrm{~Hz}$. There is an option to break down the Y-component of the traditional tympanogram and see the susceptance (B) and conductance (G) components also. In the tympanogram tab, there is also the option of viewing and printing the wide band averaged

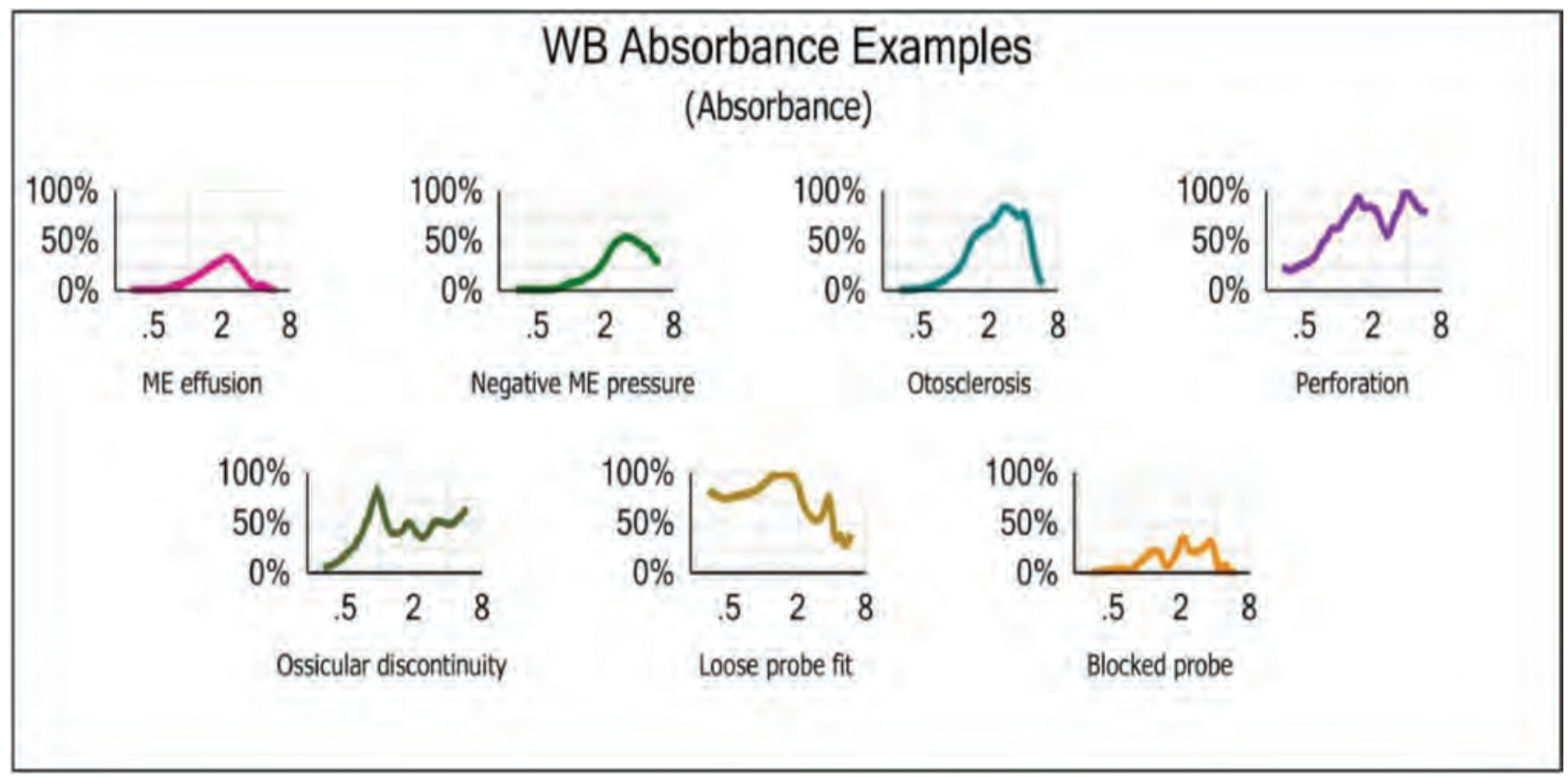

Fig. 8 Examples of the WBT absorbance graph, which have a different configuration in different diseases. Due to this, diagnosis of the nature of the middle ear pathology is a lot easier ahe clind allows a greater confidence to clinician than traditional tympanometry. 


\section{Interacoustics}

\begin{tabular}{|cr|}
\hline Patient Information: & \\
\hline Create date & Address 1 \\
Person ID & Address 2 \\
First name & City \\
Last name & Zip code \\
Birth date & Telephone \\
Gender Male & Mobile \\
\hline
\end{tabular}

\begin{tabular}{|rrr} 
Clinic: & \\
Name & VERTIGO \& DEAFNESS CLINIC & Zip coo \\
Count \\
Company Nb. & & Telephor \\
Address 1 & B] 252 Salt Lake & Fax/Mob \\
Address 2 & & Ema \\
City & KOLKATA
\end{tabular}
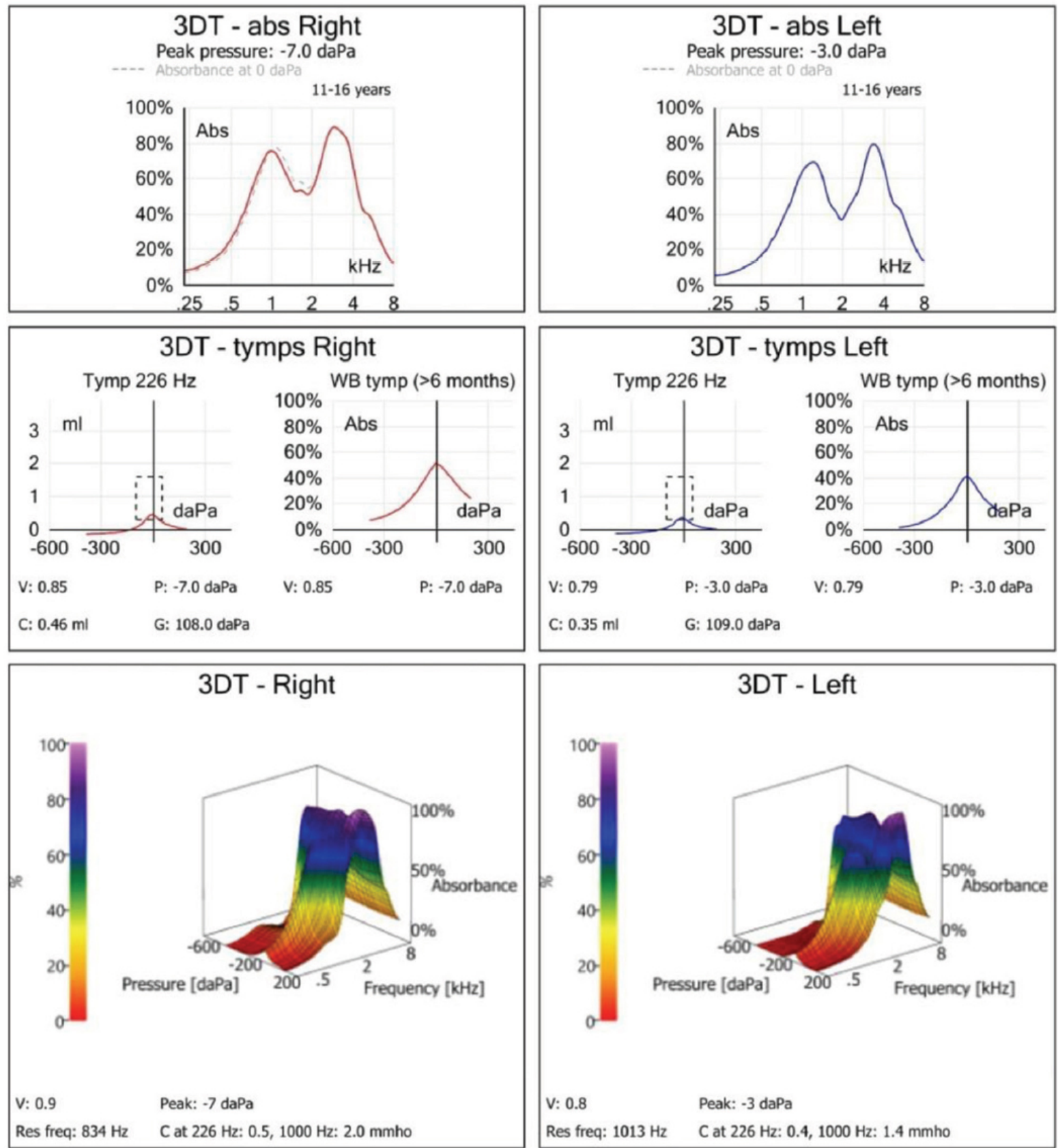

Fig. 9 Wide band tympanometry test done in the Interacoustics wide band tympanometer in the authors' clinic. The patient is a 13-year-old boy who presented with tubo-tympanic catarrh bilaterally. The test is after a course of steroids. The top graphs are the 2D absorbance graphs, in the middle are the traditional tympanograms at $226 \mathrm{~Hz}$ and $1000 \mathrm{~Hz}$, and at the bottom is the 3D composite graph. 
tympanogram for adults from 375 to $2,000 \mathrm{~Hz}$ and in infants from 800 to $2,000 \mathrm{~Hz}$ that is a much better and more accurate detector of middle ear pathology as compared with the traditional tympanogram. In the 2D WBT tab, there is also an option of knowing the resonant frequency of the middle ear that is the frequency at which most energy passes through the middle ear. There are different tabs in the screen, and the operator just has to select the output desired.

The beauty of the WBT is the absorbance graph (-Figs. 6,7 ) in which the x-axis shows the frequency and the $y$-axis shows the absorbance, that is, the acoustic energy passing through the middle ear. There is a gray area in the graph, which is the normative range, and the line graph is the absorbance plotted as a function of the frequency. This absorbance graph gives a lot of new information as explained above, but in addition, it has a typical configuration for different diseases and in different stages of the same disease like in otosclerosis ( - Fig. 8). This is a great help in diagnosis.

\section{Advantages of Wideband Tympanometry}

1. Multiple evaluations of the middle ear status and function from a single test each of which has its own and unique diagnostic value.

2. The wide band averaged tympanogram that is the composite picture of the middle ear conductance function over a wide range of frequencies is a much more sensitive and accurate test of middle ear function as compared with traditional single-frequency tympanometry.

3. There is much less of artifacts and a clearer and cleaner tympanogram recording that is free from noise and artifacts by the averaged WBT. Hence decision making is easier and ambiguity is much lesser.

4. WBT is a much better and more accurate diagnostic tool for detecting fluid in the middle ear not only in children but also in adults. This is especially useful in type $\mathrm{C}$ tympanograms where the presence of fluid is often missed by the traditional tympanometry.

5. In adults also, diseases such as superior semicircular canal dehiscence (SSCD) and ossicular discontinuity are much more confirmatively diagnosed by WBT as the absorbance graphs are very typical of these diseases. In SSCD there is increased absorbance of around $1,000 \mathrm{~Hz}$, which is evident as a peak at around $1,000 \mathrm{~Hz}$ in the absorbance graph.

6. Evaluation of the middle ear condition in ears with a perforation or a patent grommet is much better with WBT; this is not possible with traditional tympanometry.

7. The progress of disorders such as otosclerosis can be monitored by the absorbance WBT test.

8. There is also an option of doing an absorbance tympanometry at atmospheric pressure without changing the middle ear pressure. This facility can be used for monitoring middle ear function immediately after middle ear surgery when pressure changes are contraindicated.

Everything taken together, WBT is a boon to the clinical otologist, and clinicians practicing in otology should imbibe this new modality for better diagnosis and more accurate evaluation of middle ear function. The benefits of this new system of tympanometry will be realized and the test will be popularized only if practicing otologists order this test for their patients with suspected middle ear disorders.

The authors use the interacoustics Titan WBT in their Vertigo and Deafness Clinic in Kolkata and are amused with the diagnostic possibilities of this new tool ( - Fig. 9). If we are allowed to quote from the Skoda ad the WBT is "simply clever." Most limitations of traditional tympanometry can be overcome with this new innovation in tympanometry. Absorbance graphs add a new dimension to tympanometry and are a great diagnostic help. However, because the absorbance measurement is a new modality, more research and extensive use are necessary to realize its full diagnostic potentials.

\section{Suggested Reading}

1 Aithal S, Aithal V, Kei J. Effect of ear canal pressure and age on wideband absorbance in young infants. Int J Audiol 2017;56(5):346-355

2 Aithal S, Aithal V, Kei J, Andreson S. Comparison of pressurised and non-pressurised wideband absorbance in children with and without middle ear effusion. 2016

3 Baldwin M. Choice of probe tone and classification of trace patterns in tympanometry undertaken in early infancy. Int J Audiol 2006;45(7):417-427

4 Beers AN, Shahnaz N, Westerberg BD, Kozak FK. Wideband reflectance in normal Caucasian and Chinese school-aged children and in children with otitis media with effusion. Ear Hear 2010;31(2):221-233

5 Biswas A. Impedance audiometry. In: Clinical Audiovestibulometry. 5th ed. Mumbai, India: Bhalani Medical Book House; 2017:110-113

6 Expand your knowledge. Available at: www.Interacoustics. com/academy

7 Merchant GR, Röösli C, Niesten MEF, et al. Power reflectance as a screening tool for the diagnosis of superior semicircular canal dehiscence. otology \& neurotology: Official Publication of the American Otological Society, American Neurotology Society. Eur Acad Otol Neurotol 2015;36(1):172-177

8 Polat Z, Baş B, Hayır D, Bulut E, Ataş A. Wideband tympanometry normative data for Turkish young adult population. J Int Adv Otol 2015;11(2):157-162

9 Clinical applications of wideband tympanometry. Korean J Otorhinolaryngol-Head Neck Surg 2017;60(8):375-380

10 Liu YW, Sanford CA, Ellison JC, Fitzpatrick DF, Gorga MP, Keefe DH. Wideband absorbance tympanometry using pressure sweeps: system development and results on adults with normal hearing. The Journal of the Acoustical Society of America 2008;124(6). doi: $10.1121 / 1.3001712$ 